\title{
Gradient flow equation in SQCD
}

\author{
Daisuke Kadoh*† \\ Physics Division, National Center for Theoretical Sciences, National Tsing-Hua University, \\ Hsinchu, 30013, Taiwan; Research and Educational Center for Natural Sciences, Keio \\ University, Yokohama 223-8521, Japan \\ E-mail: kadoh@keio.jp, kado@cts.nthu.edu.tw
}

\section{Naoya Ukita}

Center for Computational Sciences, University of Tsukuba, Tsukuba, Ibaraki 305-8577, Japan

E-mail: ukita@ccs.tsukuba.ac.jp

We propose a supersymmetric gradient flow in $\mathscr{N}=1 \mathrm{SQCD}$ in four dimensions. The flow equation is derived in the superfield formalism and is also given for component fields of the Wess-Zumino gauge in a gauge covariant manner. We find that the flow for the component fields is supersymmetric in a sense that the flow time derivative and any supersymmetry transformation commute with each other up to a gauge transformation.

37th International Symposium on Lattice Field Theory - Lattice2019

16-22 June 2019

Wuhan, China

\footnotetext{
* Speaker.

${ }^{\dagger}$ This work is supported by JSPS KAKENHI Grant JP19K03853.
} 


\section{Introduction}

Gradient flow is widely accepted as a useful tool in various fields of physics. In lattice gauge theory, the Yang-Mills flow [1, 2, 3] leads to many interesting applications such as a construction of energy-momentum tensor on a lattice $[4,5]$, based on the finiteness of flowed field correlators [2]. So, if a gradient flow approach is extended to SUSY cases, further interesting researches could be created.

For $\mathscr{N}=1 \mathrm{SYM}$, a non-SUSY flow is defined by the YM flow [1] and a flow for a gaugino [3], which has been studied in $[6,7,8]$. On the other hand, a SUSY flow is defined by the gradient of the SYM action with respect to a vector superfield [9]. The latter flow can be given for the component fields in a gauge covariant and supersymmetric manner [10], and the finiteness of flowed field correlators can be shown for the whole gauge supermultiplet [11]. ${ }^{1}$

In this paper, a SUSY gradient flow is derived for $\mathscr{N}=1 \mathrm{SQCD}$ in four dimensions. For the gauge multiplet, a flow equation is defined in the similar manner as that of SYM while, for the matter multiplet, a technical modification is needed to define a SUSY flow in the superfield formalism. The flow can also be written in the component fields by taking the Wess-Zumino gauge, which has the SUSY covariance since the flow time and supersymmetry commute with each other up to a gauge transformation.

\section{SQCD in the superfield formalism}

The $\mathscr{N}=1 \mathrm{SQCD}$ is a gauge theory that consists of a gauge multiplet $\left(A_{\mu}, \lambda, D\right)$ and $N_{f}$ matter multiplets $\left(\phi_{ \pm}, q_{ \pm}, F_{ \pm}\right)_{f}$ for $f=1,2, \ldots, N_{f}$. Here $A_{\mu}$ is a gauge field, $\lambda_{\alpha},\left(q_{ \pm}\right)_{\alpha}$ are twocomponent spinors, $D, F_{ \pm}$are real and complex auxiliary fields, respectively. The gauge group is $S U(N)$ (or $U(N)$ ) and the generators $T^{a}$ are hermitian matrices normalized $\operatorname{as} \operatorname{tr}\left(T^{a} T^{b}\right)=\frac{1}{2} \delta_{a b}$. Although the gradient flow is a kind of diffusion equation in Euclidean spacetime, we derive it in Minkowski spacetime with the superfield formalism. The euclidean theory is obtained by the Wick rotation $t \rightarrow-i t, A_{0} \rightarrow i A_{0}$ and replacements of the auxiliary fields $D \rightarrow i D, F_{ \pm} \rightarrow i F_{ \pm}$ and $F_{ \pm}^{\dagger} \rightarrow i F_{ \pm}^{\dagger}$. We consider the massless $N_{f}=1$ case for simplicity of explanation, and it is straightforward to extend our results to a case of multi flavors with a non zero mass term.

The action of $\mathscr{N}=1 \mathrm{SQCD}$ in Minkowski space is given by $S_{\mathrm{SQCD}}=S_{\mathrm{SYM}}+S_{\mathrm{MAT}}:^{2}$

$$
\begin{aligned}
S_{\mathrm{SYM}}=\frac{1}{g^{2}} \int d^{4} x \operatorname{tr}\left\{-\frac{1}{2} F_{\mu \nu}^{2}-2 i \bar{\lambda} \bar{\sigma}^{\mu} D_{\mu} \lambda+D^{2}\right\}, \\
S_{\mathrm{MAT}}=\int d^{4} x\left\{-\left|D_{\mu} \phi_{+}\right|^{2}-\left|D_{\mu} \phi_{-}\right|^{2}+\left|F_{+}\right|^{2}+\left|F_{-}\right|^{2}\right. \\
\quad-i \bar{q}_{+} \bar{\sigma}^{\mu} D_{\mu} q_{+}-i q_{-} \sigma^{\mu} D_{\mu} \bar{q}_{-}+\phi_{+}^{\dagger} D \phi_{+}-\phi_{-} D \phi_{-}^{\dagger} \\
\left.+\sqrt{2} i\left(\phi_{+}^{\dagger} \lambda q_{+}+\phi_{-} \bar{\lambda} \bar{q}_{-}-\bar{q}_{+} \bar{\lambda} \phi_{+}-q_{-} \lambda \phi_{-}^{\dagger}\right)\right\},
\end{aligned}
$$

\footnotetext{
${ }^{1}$ See [12] for the SUSY gradient flow in the Wess-Zumino model.

${ }^{2}$ We basically follow the notation used in Ref. [13]. The Greek indices $\mu, v, \rho, \sigma$ run from 0 to 3 . The metric is given by $\eta_{\mu v}=\operatorname{diag}\{-1,+1,+1,+1\}$. The four component $\left(\sigma^{\mu}\right)_{\alpha \dot{\beta}}$ and $\left(\bar{\sigma}^{\mu}\right)^{\dot{\alpha} \beta}$ are defined as $\sigma^{\mu}=\left(-i, \sigma^{i}\right)$ and $\bar{\sigma}=\left(-1,-\sigma^{i}\right)$ where $\sigma^{i}$ is the standard Pauli matrix. We use $\sigma^{\mu v} \equiv \frac{1}{4}\left(\sigma^{\mu} \bar{\sigma}^{v}-\sigma^{v} \bar{\sigma}^{\mu}\right)$. See [13] for other details of the notation and useful identities for two-component spinors and $\sigma^{\mu}$ matrix.
} 
where $F_{\mu \nu}=\partial_{\mu} A_{v}-\partial_{v} A_{\mu}+i\left[A_{\mu}, A_{v}\right]$ and $D_{\mu}$ is a covariant derivative in the representation of acting fields: $D_{\mu} \varphi=\partial_{\mu} \varphi+i\left[A_{\mu}, \varphi\right]$ for $\varphi=\lambda, D, F_{\rho \sigma}$ and $D_{\mu} X=\partial_{\mu} X+i A_{\mu} X$ for $X=\phi_{+}, q_{+}$, $F_{+}, \phi_{-}^{\dagger}, \bar{q}_{-}, F_{-}^{\dagger}$. The action is invariant under an infinitesimal gauge transformation,

$$
\delta_{\omega}^{g} A_{\mu}=-D_{\mu} \omega, \quad \delta_{\omega}^{g} \varphi=i[\omega, \varphi], \quad \delta_{\omega}^{g} X=i \omega X,
$$

and a SUSY transformation,

$$
\begin{aligned}
& \delta_{\xi} A_{\mu}=i \xi \sigma_{\mu} \bar{\lambda}+i \bar{\xi} \bar{\sigma}_{\mu} \lambda, \\
& \delta_{\xi} \lambda=\sigma^{\mu v} \xi F_{\mu v}+i \xi D \\
& \delta_{\xi} D=-\xi \sigma_{\mu} D_{\mu} \bar{\lambda}+\bar{\xi} \bar{\sigma}_{\mu} D_{\mu} \lambda \\
& \delta_{\xi} \phi_{ \pm}=\sqrt{2} \xi q_{ \pm}, \\
& \delta_{\xi} q_{ \pm}=\sqrt{2} i \sigma^{\mu} \bar{\xi} D_{\mu} \phi_{ \pm}+\sqrt{2} \xi F_{ \pm}, \\
& \delta_{\xi} F_{+}=\sqrt{2} i \bar{\xi} \bar{\sigma}^{\mu} D_{\mu} q_{+}+2 i \bar{\xi} \bar{\lambda} \phi_{+}, \\
& \delta_{\xi} F_{-}=\sqrt{2} i \bar{\xi} \bar{\sigma}^{\mu} D_{\mu} q_{-}-2 i \phi_{-} \bar{\xi} \bar{\lambda},
\end{aligned}
$$

where $\xi_{\alpha}$ is a global anti-commuting parameter.

In the superfield formalism, a superfield $F$ is introduced as a function of $z=\left(x^{\mu}, \theta_{\alpha}, \bar{\theta}_{\dot{\alpha}}\right)$ which transforms under a supersymmetry transformation as

$$
\delta F(z)=(\xi Q+\bar{\xi} \bar{Q}) F(z)
$$

where $\theta_{\alpha}, \bar{\theta}_{\dot{\alpha}}$ are two component anti-commuting parameters, and $Q_{\alpha}$ and $\bar{Q}_{\dot{\alpha}}$ are differential operators defined by

$$
Q_{\alpha}=\frac{\partial}{\partial \theta^{\alpha}}-i\left(\sigma^{\mu}\right)_{\alpha \dot{\alpha}} \bar{\theta}^{\dot{\alpha}} \partial_{\mu}, \quad \bar{Q}_{\dot{\alpha}}=-\frac{\partial}{\partial \bar{\theta}^{\dot{\alpha}}}+i \theta^{\alpha}\left(\sigma^{\mu}\right)_{\alpha \dot{\alpha}} \partial_{\mu},
$$

For later use, let us introduce other differential operators $D_{\alpha}$ and $\bar{D}_{\dot{\alpha}}$ as

$$
D_{\alpha}=\frac{\partial}{\partial \theta^{\alpha}}+i\left(\sigma^{\mu}\right)_{\alpha \dot{\alpha}} \bar{\theta}^{\dot{\alpha}} \partial_{\mu}, \quad \bar{D}_{\dot{\alpha}}=-\frac{\partial}{\partial \bar{\theta}^{\dot{\alpha}}}-i \theta^{\alpha}\left(\sigma^{\mu}\right)_{\alpha \dot{\alpha}} \partial_{\mu} .
$$

Note that $\left\{Q_{\alpha}, \bar{Q}_{\dot{\beta}}\right\}=-\left\{D_{\alpha}, \bar{D}_{\dot{\beta}}\right\}=2 i \sigma_{\alpha \dot{\beta}}^{\mu} \partial_{\mu}$ and the other anti-commutation relations are zero.

A superfield $\Lambda$ with a superchiral condition $\bar{D}_{\dot{\alpha}} \Lambda=0$ is called a chiral superfield and may be expanded as

$$
\Lambda(y, \theta)=A(y)+\sqrt{2} \theta \psi(y)+\theta \theta F(y)
$$

with $y^{\mu}=x^{\mu}+i \theta \sigma^{\mu} \bar{\theta}$. Similarly, an anti-chiral superfield $\Lambda^{\dagger}$ is defined by $D_{\alpha} \Lambda^{\dagger}=0$. A superfield $V$ that satisfies $V=V^{\dagger}$ is called a vector superfield, which is expanded as

$$
\begin{array}{r}
V(x, \theta, \bar{\theta})=\frac{1}{2} C(x)+i \theta \chi(x)+\frac{i}{2} \theta \theta(M(x)+i N(x))-\frac{1}{2} \theta \sigma^{\mu} \bar{\theta} A_{\mu}(x) \\
+i \theta \theta \bar{\theta}\left(\bar{\lambda}(x)+\frac{i}{2} \bar{\sigma}^{\mu} \partial_{\mu} \chi(x)\right)+\frac{1}{4} \theta \theta \bar{\theta} \bar{\theta}\left(D(x)+\frac{1}{2} \square C(x)\right)+\text { h.c. }
\end{array}
$$

where $C, M, N, A_{\mu}, D$ are bosonic fields and $\chi, \lambda$ are fermionic fields. 
The SQCD action is then written as

$$
\begin{aligned}
& S_{S Y M}=\frac{1}{2 g^{2}} \int d^{4} x \operatorname{tr}\left(\left.W^{\alpha} W_{\alpha}\right|_{\theta \theta}+\text { h.c. }\right), \\
& S_{\mathrm{MAT}}=\left.\int d^{4} x\left\{Q_{+}^{\dagger} e^{2 V} Q_{+}+Q_{-} e^{-2 V} Q_{-}^{\dagger}\right\}\right|_{\theta \theta \bar{\theta} \bar{\theta}},
\end{aligned}
$$

where

$$
W_{\alpha}=-\frac{1}{8} \bar{D} \bar{D} e^{-2 V} D_{\alpha} e^{2 V}
$$

with $V=\sum_{a=1}^{N_{c}^{2}-1} V^{a} T^{a}$, and $Q_{ \pm}$are chiral superfields,

$$
Q_{ \pm}(y, \theta)=\phi_{ \pm}(y)+\sqrt{2} \theta q_{ \pm}(y)+\theta \theta F_{ \pm}(y) .
$$

The superfield action has unwanted $C, \chi, M, N$ fields which are not included in eqs. (2.1) and (2.2) with an enlarged symmetry. Eqs. (2.10) and (2.11) are actually invariant under SUSY transformation eq. (2.5) and an extended gauge transformation generated by a chiral superfield $\Lambda$ as

$$
\begin{aligned}
& e^{2 V} \rightarrow e^{2 V^{\prime}}=e^{2 \Lambda^{\dagger}} e^{2 V} e^{2 \Lambda}, \\
& Q_{+} \rightarrow Q_{+}^{\prime}=e^{-2 \Lambda} Q_{+}, \\
& Q_{-} \rightarrow Q_{-}^{\prime}=Q_{-} e^{2 \Lambda} .
\end{aligned}
$$

It is possible to remove the extra $C, \chi, M, N$ fields by choosing the component fields of $\Lambda$ by hand so that

$$
C=\chi=M=N=0,
$$

which is called the Wess-Zumino gauge. With this gauge fixing, Eqs.(2.1) and (2.2) are reproduced from the superfield action eqs. (2.10) and (2.11). Then the gauge and SUSY transformations, eqs. (2.3) and (2.4), are also reproduced as part of enlarged symmetry keeping this gauge.

\section{Derivation of SQCD flow}

In order to define a supersymmetric gradient flow, the vector and chiral superfields are transcribed into $t$-dependent superfields where $t$ is a flow time with $V(z, t=0)=V(z)$ and $Q_{ \pm}(z, t)=$ $Q_{ \pm}(z)$ at $t=0$. We assume that all properties of superfields are inherited into the $t$-dependent superfields while $\theta$ and $\bar{\theta}$ are $t$-independent. For instance, supersymmetry transformation is given by $\delta F(z, t)=(\xi Q+\bar{\xi} \bar{Q}) F(z, t)$ where $\xi$ and $\bar{\xi}$ are $t$-independent and $Q_{\alpha}, \bar{Q}_{\dot{\alpha}}$ defined by eq. (2.6) are kept unchanged. In the following, the superfield action eqs. (2.10) and (2.11) are given by replacing $V(z) \rightarrow V(z, t), Q_{ \pm}(z) \rightarrow Q_{ \pm}(z, t)$.

For the gauge multiplet, a supersymmetric gradient flow is defined as

$$
\partial_{t} V^{a}=-\frac{1}{2} g^{a b} \frac{\delta S_{\mathrm{SQCD}}}{\delta V^{b}},
$$

where $g_{a b}(V)$ is a metric derived from a norm,

$$
\|\delta V\|^{2}=\frac{1}{2} \int d^{8} z \operatorname{tr}\left(e^{-2 V} \delta e^{2 V} e^{-2 V} \delta e^{2 V}\right)(z),
$$


such that $\|\delta V\|^{2}=\int d^{8} z g_{a b}(V) \delta V^{a} \delta V^{b}$. Without the metric, the RHS and the LHS of eq.(3.1) obey different transformation laws under the extended gauge transformation which is nonlinear as in the case of general relativity. Since this norm is invariant under both of the $t$-independent super and extended gauge transformations, the flow equation with the metric eq. (3.1) is covariant under these two transformations.

Let us consider a supersymmetric flow for the matter multiplets. A naive one for $Q_{+}$would be given by $\partial_{t} Q_{+}=\delta S_{\mathrm{SQCD}} / \delta Q_{+}^{\dagger}$. This is however not a correct flow equation because the superchiral condition $\left(\bar{D}_{\dot{\alpha}} Q_{+}=0\right)$ is not kept and the RHS is not proportional to $\square Q_{+}$in the free limit as

$$
\frac{\delta S_{\mathrm{SQCD}}}{\delta Q_{+}^{\dagger}}=-\frac{1}{4} D D\left(e^{2 V} Q_{+}\right),
$$

where the functional derivative is one keeping the superchiral condition (See the derivation of field equations in Ref. [13]). If the RHS of eq. (3.3) is multiplied by $\bar{D}^{2}$, the superchiral condition is kept since $\bar{D}_{\dot{\alpha}} \bar{D}^{2}=0$, and $\square Q_{+}$term appears as $\bar{D}^{2} D^{2}=-4 \square$. Therefore a SUSY flow for the matter multiplets may be given by

$$
\begin{aligned}
& \partial_{t} Q_{+}=-\frac{1}{4} \bar{D} \bar{D}\left(e^{-2 V} \frac{\delta S_{\mathrm{MAT}}}{\delta Q_{+}^{\dagger}}\right) \\
& \partial_{t} Q_{-}=-\frac{1}{4} \bar{D} \bar{D}\left(\frac{\delta S_{\mathrm{MAT}}}{\delta Q_{-}^{\dagger}} e^{2 V}\right) .
\end{aligned}
$$

Note that $S_{\mathrm{MAT}}$ is the same as $S_{\mathrm{SQCD}}$ under $\delta / \delta Q$ since $S_{\mathrm{SYM}}$ does not have $Q_{ \pm}$. These equations are covariant under $t$-independent super and extended gauge transformations. We thus find that SQCD flow equations are defined by eqs. (3.1), (3.4), (3.4) in the superfield formalism.

These flow equations are modified to define SUSY flows for the component fields of the WessZumino gauge. The straightforward calculations tell us that the Wess-Zumino gauge eq.(2.15) is not kept after the time evolution because $\partial_{t} C=-D-\left(\phi_{+}^{\dagger} T^{a} \phi_{+}-\phi_{-} T^{a} \phi_{-}^{\dagger}\right) T^{a} \neq 0$ and the RHS of $\partial_{t} \chi, \partial_{t} M, \partial_{t} N$ are also non zero. To keep the Wess-Zumino gauge, we modify the SUSY flow equations adding an extended gauge transformation as

$$
\begin{aligned}
& \partial_{t} V^{a}=-\frac{1}{2} g^{a b} \frac{\delta S_{\mathrm{SQCD}}}{\delta V^{b}}+\delta_{\Lambda} V^{a} \\
& \partial_{t} Q_{+}=-\frac{1}{4} \bar{D} \bar{D}\left(e^{-2 V} \frac{\partial S_{S Q C D}}{\partial Q_{+}^{\dagger}}\right)+\delta_{\Lambda} Q_{+} \\
& \partial_{t} Q_{-}=-\frac{1}{4} \bar{D} \bar{D}\left(\frac{\partial S_{S Q C D}}{\partial Q_{-}^{\dagger}} e^{2 V}\right)+\delta_{\Lambda} Q_{-},
\end{aligned}
$$

where $\delta_{\Lambda}$ is an infinitesimal transformation derived from eq. (2.14). We take component fields of $\Lambda$ by hand such that $\partial_{t} C=\partial_{t} \chi=\partial_{t} M=\partial_{t} N=0$ :

$$
\begin{aligned}
A & =\frac{D}{2}+\frac{1}{2}\left(\phi_{+}^{\dagger} T^{a} \phi_{+}-\phi_{-} T^{a} \phi_{-}^{\dagger}\right) T^{a}, \\
\psi & =-\frac{1}{\sqrt{2}} \sigma^{\mu} D_{\mu} \bar{\lambda}+\left(\phi_{+}^{\dagger} T^{a} q_{+}-q_{-} T^{a} \phi_{-}^{\dagger}\right) T^{a}, \\
F & =\left(\phi_{+}^{\dagger} T^{a} F_{+}-F_{-} T^{a} \phi_{-}^{\dagger}\right) T^{a} .
\end{aligned}
$$


Using these $A, \psi, F$, the Wess-Zumino gauge fixing is maintained for any nonzero flow time as long as it is set at $t=0$.

Thus we obtain the flow equations of the components fields: for the gauge multiplet,

$$
\begin{aligned}
\partial_{t} A_{\mu}= & D^{\rho} F_{\rho \mu}-\lambda \sigma_{\mu} \bar{\lambda}-\bar{\lambda} \bar{\sigma}_{\mu} \lambda+i\left(\phi_{+}^{\dagger} T^{a} D_{\mu} \phi_{+}-D_{\mu} \phi_{-} T^{a} \phi_{-}^{\dagger}-h . c .\right) T^{a} \\
& +\left(\bar{q}_{+} \bar{\sigma}^{\mu} T^{a} q_{+}+q_{-} \sigma_{\mu} T^{a} \bar{q}_{-}\right) T^{a} \\
\partial_{t} \lambda= & -\sigma^{\mu} \bar{\sigma}^{v} D_{\mu} D_{v} \lambda-[\lambda, D]-\sqrt{2} \sigma^{\mu}\left(\bar{q}_{+} T^{a} D_{\mu} \phi_{+}-D_{\mu} \phi_{-} T^{a} \bar{q}_{-}\right) T^{a} \\
& -\sqrt{2} i\left(F_{+}^{\dagger} T^{a} q_{+}-q_{-} T^{a} F_{-}^{\dagger}\right) T^{a}-\left(\phi_{+}^{\dagger} \lambda T^{a} \phi_{+}+\phi_{-} \bar{\lambda} T^{a} \phi_{-}^{\dagger}+h . c .\right) T^{a} \\
\partial_{t} D= & D^{\mu} D_{\mu} D+i\left(D_{\mu} \lambda \sigma^{\mu} \bar{\lambda}-D_{\mu} \bar{\lambda} \bar{\sigma}^{\mu} \lambda-h . c .\right)-\left(\phi_{+}^{\dagger} D T^{a} \phi_{+}+\phi_{-} D T^{a} \phi_{-}^{\dagger}+h . c .\right) T^{a} \\
& +2\left(D^{\mu} \phi_{+}^{\dagger} T^{a} D_{\mu} \phi_{+}-D_{\mu} \phi_{-} T^{a} D_{\mu} \phi_{-}^{\dagger}\right) T^{a}+2 \sqrt{2} i\left(\bar{q}_{+} T^{a} \bar{\lambda} \phi_{+}+\phi_{-} \bar{\lambda} T^{a} \bar{q}_{-}-h . c .\right) T^{a} \\
& +i\left(\bar{q}_{+} T^{a} \bar{\sigma}^{\mu} D_{\mu} q_{+}-q_{-} T^{a} \sigma^{\mu} D_{\mu} \phi_{-}^{\dagger}-h . c .\right) T^{a}-2\left(F_{+}^{\dagger} T^{a} F_{+}-F_{-} T^{a} F_{-}^{\dagger}\right) T^{a},
\end{aligned}
$$

and, for the matter multiplet,

$$
\begin{aligned}
\partial_{t} \phi_{+}= & D^{\mu} D_{\mu} \phi_{+}+i \sqrt{2} \lambda q_{+}-\left(\phi_{+}^{\dagger} T^{a} \phi_{+}-\phi_{-} T^{a} \phi_{-}^{\dagger}\right) T^{a} \phi_{+}, \\
\partial_{t} q_{+}= & -\sigma^{\mu} \bar{\sigma}^{v} D_{\mu} D_{v} q_{+}+i \sqrt{2} \lambda F_{+}-\sqrt{2} \sigma^{\mu} \bar{\lambda} D_{\mu} \phi_{+}-D q_{+} \\
& -\left(\phi_{+}^{\dagger} T^{a} \phi_{+}-\phi_{-} T^{a} \phi_{-}^{\dagger}\right) T^{a} q_{+}-2\left(\phi_{+}^{\dagger} T^{a} q_{+}-q_{-} T^{a} \phi_{-}^{\dagger}\right) T^{a} \phi_{+}, \\
\partial_{t} F_{+}= & D^{\mu} D_{\mu} F_{+}-2 D F_{+}+\sqrt{2}\left(D_{\mu} \bar{\lambda} \bar{\sigma}^{\mu} q_{+}-\bar{\lambda} \bar{\sigma}^{\mu} D_{\mu} q_{+}\right)-2 \bar{\lambda} \bar{\lambda} \phi_{+} \\
& -\left(\phi_{+}^{\dagger} T^{a} \phi_{+}-\phi_{-} T^{a} \phi_{-}^{\dagger}\right) T^{a} F_{+}+2\left(\phi_{+}^{\dagger} T^{a} q_{+}-q_{-} T^{a} \phi_{-}^{\dagger}\right) T^{a} q_{+} \\
& -2\left(\phi_{+}^{\dagger} T^{a} F_{+}-F_{-} T^{a} \phi_{-}^{\dagger}\right) T^{a} \phi_{+},
\end{aligned}
$$

where similar equations are given for $\phi_{-}, q_{-}, F_{-}$. Note that we are now working on the Minkowski space. After the Wick rotation, the euclidean flow equations, which are kinds of diffusion equations, are obtained for all fields.

These equations are gauge covariant under the $t$-independent gauge transformations. The supersymmetry transformations for the flowed fields are defined by eq. (2.4) replacing all of the fields with the corresponding $t$-dependent fields. Then one can show that

$$
\left[\partial_{t}, \delta_{\xi}\right]=\delta_{\omega}^{g}, \quad \omega \equiv-i D_{\mu}\left(\xi \sigma_{\mu} \bar{\lambda}+\bar{\xi} \bar{\sigma}_{\mu} \lambda\right),
$$

where $\delta_{\omega}^{g}$ is an infinitesimal gauge transformations with a gauge parameter $\omega$, which is given by eq. (2.3). The obtained flows are supersymmetric since the RHS of eq. (3.14) vanishes for gauge invariant quantities.

These flow equations can be simplified without breaking the supersymmetry covariance. The gradient of $S_{\mathrm{SYM}}$ is also employed to define a flow for the gauge multiplet in eq. (3.1) instead of $S_{\mathrm{SQCD}}$. Although the flows for the matter multiplet are given by the same expressions as eqs. (3.4) and (3.5), this change affects the whole of flow equations for the component fields because $\Lambda$ changes. A straightforward calculation yields

$$
\begin{aligned}
& \partial_{t} A_{\mu}=D^{\rho} F_{\rho \mu}-\lambda \sigma_{\mu} \bar{\lambda}-\bar{\lambda} \bar{\sigma}_{\mu} \lambda, \\
& \partial_{t} \lambda=-\sigma^{\mu} \bar{\sigma}^{v} D_{\mu} D_{v} \lambda-[\lambda, D], \\
& \partial_{t} D=D^{\mu} D_{\mu} D+i\left(D_{\mu} \lambda \sigma^{\mu} \bar{\lambda}-D_{\mu} \bar{\lambda} \bar{\sigma}^{\mu} \lambda-\text { h.c. }\right),
\end{aligned}
$$


and

$$
\begin{aligned}
& \partial_{t} \phi_{+}=D^{\mu} D_{\mu} \phi_{+}+i \sqrt{2} \lambda q_{+}, \\
& \partial_{t} q_{+}=-\sigma^{\mu} \bar{\sigma}^{v} D_{\mu} D_{v} q_{+}+i \sqrt{2} \lambda F_{+}-\sqrt{2} \sigma^{\mu} \bar{\lambda} D_{\mu} \phi_{+}-D q_{+}, \\
& \partial_{t} F_{+}=D^{\mu} D_{\mu} F_{+}-2 D F_{+}+\sqrt{2}\left(D_{\mu} \bar{\lambda} \bar{\sigma}^{\mu} q_{+}-\bar{\lambda} \bar{\sigma}^{\mu} D_{\mu} q_{+}\right)-2 \bar{\lambda} \bar{\lambda} \phi_{+} .
\end{aligned}
$$

where similar equations are given for $\phi_{-}, q_{-}, F_{-}$. These simplified flows also satisfy eq. (3.14).

\section{Summary and future outlook}

We have derived a supersymmetric flow equation in four-dimensional $\mathscr{N}=1$ SQCD using the superfield formalism. The flows can also be written in the component fields of the WessZumino gauge in a gauge covariant manner. We have directly confirmed that the obtained flow is supersymmetric in a sense that the flow time derivative and supersymmetry commute with each other up to a gauge transformation at any non-zero flow time.

As a next step of our research, we are investigating the finiteness of flowed field correlators at all order of the perturbation theory. Then we aim to construct the Ferrara-Zumino multiplet on the lattice using a small flow time expansion of flowed field correlators, after which we will use our method in actual numerical simulations to study Seiberg duality in $\mathscr{N}=1$ SQCD.

SUSY flows of $\mathscr{N}=2$ and $\mathscr{N}=4$ theories would be derived using similar techniques given in this paper. If they are given in terms of $\mathscr{N}=1$ superfield formalism, eq. (3.14) would not be satisfied for the whole of extended supersymmetry. In the case, it is not obvious how to hold the finiteness of flowed field correlators. Therefore further studies are needed to understand SUSY flows in extended SUSY theories.

\section{References}

[1] M. Lüscher, JHEP 1008 (2010) 071 Erratum: [JHEP 1403 (2014) 092].

[2] M. Lüscher and P. Weisz, JHEP 1102 (2011) 051.

[3] M. Lüscher, JHEP 1304 (2013) 123.

[4] H. Suzuki, PTEP 2013 (2013) 083B03 Erratum: [PTEP 2015 (2015) 079201].

[5] H. Makino and H. Suzuki, PTEP 2014 (2014) 063B02 Erratum: [PTEP 2015 (2015) 079202].

[6] K. Hieda, A. Kasai, H. Makino and H. Suzuki, PTEP 2017 (2017) no.6, $063 B 03$.

[7] A. Kasai, O. Morikawa and H. Suzuki, PTEP 2018 (2018) no.11, 113B02.

[8] G. Bergner, C. López and S. Piemonte, Phys. Rev. D 100 (2019) no.7, 074501.

[9] K. Kikuchi and T. Onogi, JHEP 1411 (2014) 094.

[10] D. Kadoh and N. Ukita, arXiv:1812.02351 [hep-th].

[11] D. Kadoh, Talk at a workshop "Quantum Gravity meets lattice QFT", ECT*, Trento, Sep 6, 2018

[12] D. Kadoh, K. Kikuchi and N. Ukita, Phys. Rev. D 100 (2019) no.1, 014501.

[13] J. Wess and J. Bagger, "Supersymmetry and Supergravity SECOND EDITION, REVISED AND EXPANDED," Princeton Serieis in Physics Princeton University Press, Princeton, New Jersey (1992). 